\title{
Análise Sistêmica de Efetividade: aplicação ao BNDES Funtec
}

Roberto de Oliveira Pereira

Guilherme Costa Pereira

Luciana Xavier de Lemos Capanema

Eduardo Pinho Pereira e Souza*
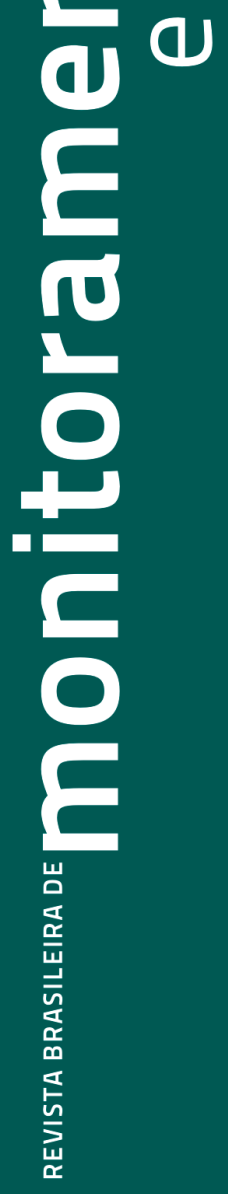

70

* Respectivamente engenheiro, economista, gerente e administrador do BNDES. O presente artigo é de exclusiva responsabilidade dos autores, não refletindo, necessariamente, a opinião do BNDES. 


\section{Resumo}

O OBJETIVO DESTE ARTIGO É APRESENTAR UMA ABORDAGEM SISTÊMICA PARA O Monitoramento e AvaliaçÃo (M\&A) de PROGRAMAS do Banco NaCIONAL dE DesenVOLVIMENTO ECONÔMICO E SOCIAL (BNDES). A EXPERIÊNCIA DO BNDES EM M\&A FOI INICIADA COM O USO DO QUADRO LÓGICO, OUE TEVE UM IMPORTANTE PAPEL NA EXPLICITAÇÃO DOS OBJETIVOS E INDICADORES DE EFETIVIDADE DOS PROGRAMAS OPERACIONALIZADOS PELO BANCO. ENTRETANTO, CONSTATOU-SE OUUE O QUUADRO LóGICO NÃO ERA ADEOUADO AOS CASOS DE PROGRAMAS MAIS COMPLEXOS, NOS OUUAS OS EFEITOS SISTÊMICOS SÃO MAIS RELEVANTES. A MOTIVAÇÃO PRINCIPAL, ENTÃO, FOI CRIAR UMA FERRAMENTA CAPAZ DE MODELAR E CAPTAR OS EFEITOS DOS PROGRAMAS DO BNDES CLASSIFICADOS COMO ESTRUTURANTES. PARA TAL, ESTE ARTIGO DESENVOLVE O CONCEITO DE ANÁLISE SISTÊMICA DE EFETIVIDADE, BASEADO NO ARCABOUÇO CONCEITUAL DO BALANCED SCORECARD E NA TEORIA DE SISTEMAS, PARA CRIAR UMA ABORDAGEM SISTÊMICA DE ANÁLISE DOS EFEITOS DE UMA POLÍTICA PÚBLICA. EM SEGUIDA, O MÉTODO FOI APLICADO AO CASO DO FUNDO TECNOLÓGICO DO BNDES (FUNTEC), UM INSTRUMENTO DE APOIO NÃO REEMBOLSÁVEL, CUJA FINALIDADE É APOIAR PROJETOS COOPERATIVOS ENTRE ACADEMIA E EMPRESAS DE PESOQUISA TECNOLÓGICA APLICADA. O MÉTODO MOSTROU-SE ADEOUADO PARA A MODELAGEM DO FUNTEC, APRESENTANDO RESULTADOS COM MAIOR RIOUUEZA DO OUE SERIA ESPERADO COM A APLICAÇÃO DO QUUADRO LÓGICO.

\section{Abstract}

THE PURPOSE OF THIS ARTICLE IS TO PRESENT A SYSTEMIC APPROACH TO MONITORING AND ASSESSING THE PROGRAMS OF THE BRAZILIAN DEVELOPMENT BANK, BNDES. THE BNDES EXPERIENCE WITH SUCH ASSESSMENT STARTED WITH THE USE OF THE LOGICAL FRAMEWORK, WHICH PLAYED AN IMPORTANT ROLE IN REVEALING THE PURPOSES AND EFFECTIVENESS INDEXES OF PROGRAMS THE BANK HAS SUPPORTED. HOWEVER, IT WAS FOUND THAT THE LOGFRAME WAS NOT APPROPRIATE FOR MORE COMPLEX PROGRAMS, IN WHICH SYSTEMIC EFFECTS ARE MORE IMPORTANT. THEREFORE, THE MAIN INCENTIVE WAS TO CREATE A TOOL THAT WAS ABLE TO SHAPE AND ENCOMPASS THE EFFECTS OF THE BNDES' SO-CALLED STRUCTURING PROGRAMS. THUS, THIS PAPER DEVELOPS THE "SYSTEMIC ANALYSIS OF EFFECTIVENESS" CONCEPT, BASED ON THE CONCEPTUAL BALANCED SCORECARD AND SYSTEMS THEORY FRAMEWORKS TO CREATE A SYSTEMIC APPROACH TO ANALYZE THE EFFECTS OF A PUBLIC POLICY. THEN, THE METHOD WAS APPLIED TO THE BNDES' TECHNOLOGICAL FUND (FUNTEC), A NON-REFUNDABLE BNDES'S INSTRUMENT, AIMING TO SUPPORT ACADEMIA AND COMPANIES COOPERATION TO DEVELOP APPLIED TECHNOLOGICAL RESEARCH. THE METHOD PROVED APPROPRIATE IN STRUCTURING FUNTEC, SHOWING RESULTS WITH MORE DETAILS THAN EXPECTED FROM THE LOGFRAME.

\section{PALAVRAS-CHAVE:}

Balanced Scorecard; BNDES; Funtec; Monitoramento e Avaliação; Inovação.

Revista Brasileira de Monitoramento e Avaliação | Número 8 | Julho-Dezembro de 2014 


\section{Introdução}

Há mais de 30 anos as instituições multilaterais de desenvolvimento vêm dedicando esforços para monitorar e avaliar suas operações de apoio ao desenvolvimento.

Esse exercício de Monitoramento e Avaliação (MசA) se intensificou, tal como se observa na atualidade, a partir da Segunda Guerra Mundial, quando os governos em todo mundo - sobretudo no Ocidente - começaram a investir maciçamente em políticas públicas e em pesquisas que dessem suporte às intervenções nas questões sociais. Em muitas instituições o monitoramento e avaliação passaram a ser parte integrante do processo de planejamento e gestão de programas e projetos, visando à maior eficiência na aplicação de recursos e à maior eficácia e efetividade nos resultados das intervenções. ${ }^{1}$

Nesse contexto, surgiram diversas ferramentas que ajudaram a modelar tais intervenções. Uma das primeiras e mais utilizadas dessas ferramentas foi o Quadro Lógico².

Pertencente à família de modelos lógicos cujo objetivo é explicitar a teoria da mudança, ou a teoria do programa, o Quadro Lógico (QL) é um instrumento de planejamento no qual são representadas as relações de causa e efeito entre atividades, produtos e serviços bem como efeitos esperados. O método explicita o que se espera com a intervenção e seleciona os indicadores que vão captar as respectivas mudanças desejadas. Assim - QL estrutura o monitoramento periódico desses indicadores, a correção de eventuais desvios durante a implantação da interven- ção e, por consequência, a sua avaliação. Em sua difusão nas práticas internacionais, esse instrumento apresentou variações de forma e nomenclatura, como Matriz de Resultados e Marco Lógico.

Alguns modelos de M\&A incorporaram, desde a fase de planejamento da intervenção até a futura avaliação, as partes interessadas (Stakeholders), por considerá-las fundamentais para seu sucesso. Assim se desenvolveu a metodologia ZOPP3, no qual a construção do QL se dá com a participação de vários interlocutores.

Mais recentemente foi desenvolvida uma metodologia que identifica que os objetivos pretendidos por uma intervenção ocorrem preponderantemente em função da mudança de comportamento dos diversos atores envolvidos. Desta forma o método considera as partes interessadas como parceiros no sucesso da intervenção. Trata-se do Outcome Mapping, ou Mapeamento de Resultados ${ }^{4}$.

O BNDES (Banco Nacional de Desenvolvimento Econômico e Social), em 2011, formalizou a estrutura responsável pela construção de um Sistema de M\&A5. Entre as atividades conduzidas desde então se destaca a utilização do QL na formulação, monitoramento, avaliação e renovação de programas. Essa metodologia foi escolhida devido a sua simplicidade na aplicação e entendimento.

A experiência do BNDES revelou, entretanto, que nos casos em que se quer captar transformações econômicas e sociais complexas 
possivelmente deve-se abrir mão da simplicidade do QL para adotar ferramentas mais potentes de M\&A. Assim, percebeu-se que a aplicabilidade da ferramenta depende da situação concreta que se quer avaliar, bem como dos objetivos e do alcance da avaliação.

O presente artigo aborda um desenvolvimento metodológico para o M\&A de intervenções complexas, aqui denominado Análise Sistêmica de Efetividade (ASE).Este texto se estrutura em cinco seções. Além desta introdução, na segunda é apresentado um rápido histórico sobre os instrumentos operacionais do BNDES e sobre a implantação do Sistema de M\&A neste Banco; na terceira são descritas as bases conceituais da ASE, bem como os respectivos procedimentos metodológicos; e na quarta seção a metodologia ASE é aplicada ao caso do Fundo Tecnológico do BNDES (Funtec), sendo apresentado na última parte deste texto suas conclusões.

\section{O aprendizado com M\&A no BNDES}

O BNDES é um dos principais instrumentos do Governo Federal para o investimento fixo na economia. Desembolsou, nos últimos 12 meses encerrados em setembro de 2014, aproximadamente 188,5 bilhões de reais no apoio às ações de desenvolvimento dos mais diferentes tipos e nos mais diferentes setores.

Dentre as intervenções apoiadas, encontram-se investimentos industriais e de infraestrutura, aquisição de máquinas e equipamentos, bens de produção, insumos e serviços, inserção internacional de empresas brasileiras, subscrição de valores mobiliários e projetos nas áreas da cultura e meio ambiente. Além disso, o BNDES está presente no apoio a projetos de inclusão social e de inclusão produtiva. Em todos esses casos, os projetos apoiados pelo Banco são orientados por prio-

1 Entende-se por efetividade a geração de efeitos ocasionada por uma intervenção, provocando mudanças quantitativas ou qualitativas na realidade. Esses efeitos podem ser alcançados em diferentes momentos no tempo, e podem ser mais ou menos influenciados por fatores externos à intervenção.

2 Pfeiffer, 2000.

3 Planejamento de Projeto Orientado por Objetivos (da sigla em alemão ZOPP). Um manual está disponível em: < http://cursos. campusvirtualsp.org/pluginfile.php/36543/mod_page/content/7/M3C5_Minguillo_2003.pdf>.

4 Earl, Carden e Smutylo, 2001.

5 A Gerência de Avaliação de Efetividade e Emprego na Área de Planejamento. Para maiores informações sobre as avaliações de efetividade realizadas pelo BNDES, acesse: <http://www.bndes.gov.br/SiteBNDES/bndes/bndes_pt/Institucional/BNDES_ Transparente/Efetividade/>.

Revista Brasileira de Monitoramento e Avaliação | Número 8 | Julho-Dezembro de 2014 
ridades e políticas transversais, como inovação, desenvolvimento regional e política de responsabilidade socioambiental.

Os clientes que podem acessar são majoritariamente pessoas jurídicas: empresas industriais, comerciais, de serviços ou do setor agropecuário, cooperativas, associações, fundações, organizações da sociedade civil de interesse público (OSCIP). Também podem ser apoiadas esferas da administração pública, principalmente as municipais e estaduais, e até mesmo pessoas físicas, como o microempreendedor ou o agricultor familiar.

As operações também podem acontecer de forma direta, ou seja, com a análise e a contratação da operação diretamente pelo BNDES, ou de forma indireta, por meio de intermediários financeiros (principalmente bancos comerciais) credenciados pelo Banco, que assumem o risco da operação.

Assim, implantar o Sistema de M\&A numa organização com essa complexidade certamente traz alguns desafios: em primeiro lugar pelo tamanho do BNDES6, em segundo por sua complexidade - como explicitado anteriormente, são projetos cujos objetivos, tipo de cliente e desenhos financeiros são bem diferentes. E em terceiro lugar por seu sistema organizacional: é necessário mudar a cultura de trabalho para incluir aspectos avaliativos de efetividade.

A primeira preocupação, então, da equipe incumbida do Sistema de M\&A foi de tornar os instrumentos operacionais do BNDES avaliá- útil para o M\&A de programas operacionais. Com ele foi possível explicitar os objetivos dessas intervenções apoiadas pelo Banco em termos de efetividade, bem como acompanhar os respectivos indicadores, que permitem sinalizar se elas estão caminhando no sentido esperado. ${ }^{8}$

O uso do QL no BNDES não é um fato isolado no cenário brasileiro. A disseminação desse instrumento pode ser verificada em documentos do TCU9 ${ }^{9}$, da Fundação Dom Cabral ${ }^{10}$, assim como do IPEA ${ }^{11}$.

Em que pese sua comprovada utilidade, isto é, da facilidade de compreensão e aplicação, a lógica sobre a qual o QL se baseia é linear e simplificadora. Em intervenções mais complexas tal linearidade não capta satisfatoriamente toda a riqueza de efeitos potenciais.

Assim, o desenvolvimento do sistema de M\&A do BNDES, a partir desse primeiro avanço (tornando os programas operacionais avaliáveis), demanda diferentes métodos capazes de captar a complexidade de diversas outras intervenções. Nesse sentido, foi elaborada a ASE, a ser detalhada a seguir.

\section{Análise Sistêmica de Efetividade (ASE)}

Os instrumentos operacionais do BNDES geram efetividades potenciais em diferentes níveis de abrangência. Assim, aqueles cuja efetividade potencial se concentra em torno de seus objetivos declarados podem ser classificados como Finalísticos. Para estes, o OQL 
parece ser suficiente para explicitar efeitos e permitir sua avaliação. Entretanto, em outros níveis a efetividade potencial vai além dos seus objetivos declarados, por ter impactos sistêmicos, ou seja, se concentra na contribuição para outros programas ou políticas. Tais instrumentos operacionais podem ser classificados como Estruturantes, que serão o foco do presente trabalho.

É possível deixar mais claro esses conceitos utilizando dois hipotéticos programas públicos: (a) programa de apoio ao microempreendedorismo em áreas de baixa renda e (b) programa de formação profissional de jovens em áreas carentes. Ambos os programas são meritórios para o apoio público e têm efeitos diretos e indiretos relevantes. A diferença está onde se concentra a efeti- vidade potencial em cada caso. No primeiro programa pode-se reconhecer seu sucesso no aumento da geração de renda na comunidade, que é seu objetivo declarado. No segundo, o objetivo declarado é o aumento da quantidade de jovens com formação profissional. O sucesso desse programa, entretanto, espraia na empregabilidade dos jovens, na queda da violência, no afastamento das drogas etc. Ou seja, a efetividade do programa seria principalmente sistêmica. Assim, a nosso ver, esse programa seria Estruturante, enquanto o primeiro seria Finalístico. Claro que essa classificação não é absoluta: tanto os programas finalísticos possuem aspectos estruturantes, como os estruturantes têm aspectos finalísticos. Então a questão é identificar em qual nível se concentra seus efeitos potenciais.

6 Em 2013, o volume de desembolsos do BNDES foi aproximadamente 2,2 vezes maior do que o desembolso do Grupo Banco Mundial (inclui BIRD, AID, IFC e RETF).

7 O BNDES executa suas intervenções por meio de linhas, fundos, produtos e programas.

8 Uma aplicação desse processo de trabalho está apresentada no BNDES Setorial n. 36, com o Programa de Apoio ao Desenvolvimento do Setor Aquícola (Proaquicultura). Há outros exemplos publicados, como no BNDES Setorial n. 33 e na Revista do BNDES n. 41, que apresentam, respectivamente, os OLLs do Programa de Apoio ao Desenvolvimento do Setor de Fármacos e do Apoio ao Setor de Acervos.

9 BRASIL, 2001.

10 Que pode ser acessado em: <http://www.gife.org.br/artigo-planejamento-e-avaliacao-de-projetos-sociais-o-marco-logico-revisitado-15629.asp>.

11 Cassiolato e Gueresi, 2010.

Revista Brasileira de Monitoramento e Avaliação | Número 8 | Julho-Dezembro de 2014 
Quando há uma maior densidade em efeitos sistêmicos, O QL não é o instrumento mais adequado para MEA justamente por se concentrar nos efeitos dos objetivos declarados. Nesses casos parece ser mais adequada uma ferramenta que permita perceber e caracterizar com mais clareza os efeitos sistêmicos e seus indicadores.

A ASE se utiliza do arcabouço conceitual do BSC para criar uma abordagem sistêmica de análise dos efeitos de uma política pública. Para explicitar claramente o sentido dessa afirmação, é necessário dividi-la em duas partes: em primeiro lugar, a abordagem sistêmica do ferramental conceitual do BSC e, em segundo lugar, a aplicação do BSC não a uma empresa ou uma organização, mas a uma política pública.

A metodologia do BSC foi desenvolvida originalmente para comunicação e gestão da estratégia empresarial. Esta metodologia permite tratar o planejamento empresarial com uma visão sistêmica, pois envolve toda a cadeia de criação de valor da empresa (nas perspectivas de competências, processos, marketing etc.) em sua estratégia. Por outro lado, tal metodologia tem a flexibilidade para ser adaptada aos órgãos da administração pública e às organizações sociais. Nesses casos as perspectivas devem ser adaptadas de acordo com os objetivos das organizações-alvo. Por exemplo, uma organização social que se dedica à formação de músicos colocaria a perspectiva financeira como a base do mapa estratégico, pois é a partir da captação de fundos que as outras dimensões podem ser desenvolvidas até o cumprimento de sua missão. Em contrapartida, no mapa de uma empresa privada, a perspectiva financeira ocupa a posição superior, pois a lucratividade é a razão de ser da empresa ${ }^{12}$.

O segundo passo metodológico consiste em aplicar o BSC a uma política pública ${ }^{13}$. Quando se trata da empresa/organização na aplicação tradicional do BSC, o sujeito do planejamento (empresa/organização) já está suficientemente definido quanto ao seu funcionamento, suas partes e seus objetivos. Diferentemente desse uso tradicional, sua aplicação para um programa ou política possibilita que o sujeito do planejamento seja ampliado da empresa para a coletividade de organizações e populações que são os públicos-alvo da referida política pública. Essa mudança requer o amparo conceitual da Teoria de Sistemas para definir quem são os sujeitos do planejamento.

A Teoria Geral de Sistemas foi proposta pelo biólogo alemão Ludwig von Bertalanffy, em trabalho publicado em 1969, intitulado General System Theory ${ }^{14}$. Baseado em várias disciplinas - Biologia, Sociologia, Psicologia, Antropologia etc. -, o conceito de Sistema Aberto descreve as ações e interações de um organismo dentro de um ambiente. Este Sistema pode ser compreendido como um conjunto de partes em constante interação e se constitui num todo sinérgico, ou seja, o todo é maior que a soma de suas partes. Assim, é orientado para determinados propósitos e está em permanente relação de interdependência com o ambiente externo. Essa interdependência deve ser entendida como a dupla capacidade de influenciar o ambiente externo e ser influenciado por ele. 
Embora o objetivo da ASE seja, tal qual no Quadro Lógico, a descrição dos objetivos da intervenção e dos efeitos desejados bem como a escolha de métricas de acompanhamento, seu foco de atenção é diferente: enquanto o OL foca na intervenção e seus efeitos, a ASE procura enxergar o sujeito sobre o qual a intervenção ocorre, em suas várias dimensões, características e funcionamento. Por isso a ASE é mais apropriada para a modelagem de intervenções estruturantes, com maior grau de complexidade e com efeitos sistêmicos. Utilizando uma metáfora da área de saúde para melhor esclarecer o conceito, a ASE procura ver não só o remédio, mas também o paciente que vai tomá-lo.

A ASE estrutura a modelagem de uma intervenção nos seguintes procedimentos:

- descreve a população-alvo como um sistema, ao definir os elementos desse sistema, suas inter-relações, insumos, produtos e suas relações com o exterior;

- descreve as características do sistema segundo as perspectivas do BSC - competências, processos e atendimento aos clientes/público-alvo;
- descreve a política ou programa como uma intervenção no funcionamento do sistema, ao explicitar seus objetivos em cada uma das perspectivas definidas na etapa anterior;

- Define indicadores para monitoramento desses objetivos.

A seguir, será apresentada a aplicação do método para o caso do Funtec, em que será possível ilustrar detalhes desses procedimentos metodológicos.

\section{Análise Sistêmica de Efetividade do Funtec}

O Funtec é um instrumento de apoio não reembolsável do BNDES, que tem por finalidade apoiar a pesquisa científica e tecnológica aplicada de projetos desenvolvidos por Instituições Tecnológicas (IT) com a interveniência de empresas. Essa parceria é fundamental, na medida em que se objetiva a geração de inovações, ou seja, que o resultado da pesquisa seja levado ao mercado.

A realização desse objetivo central é um evento de alto risco tecnológico e/ou de

\footnotetext{
12 Kaplan e Norton, 2004.

13 Kunz e Schaaf, 2011.

14 Bertalanffy, 1969.
}

Revista Brasileira de Monitoramento e Avaliação | Número 8 | Julho-Dezembro de 2014 
longo prazo de maturação, por sua própria natureza. Entretanto, mesmo que uma baixa proporção dos projetos apoiados seja efetivamente levada ao mercado, o caráter estruturante do Funtec se revela em vários aspectos: no desenvolvimento das parcerias academia-empresa; na estruturação e desenvolvimento de equipes; na modernização de laboratórios etc.

Assim, a modelagem de M\&A do Funtec utilizando o QL, por se concentrar nos objetivos declarados do programa, possivelmente não daria ênfase a esses aspectos estruturantes, tornando o Funtec um bom candidato à aplicação da metodologia ASE.

\section{DESCRIÇÃO DO SISTEMA}

O primeiro passo da ASE é a definição do sistema a ser tratado pelo Funtec, por exemplo, o Sistema de Ciência, Tecnologia e Inovação (CT\&I). É possível caracterizar o Sistema de CT\&I como sendo composto por um conjunto de Universidades, Centros de Pesquisa públicos e privados, e os órgãos de administração pública relacionados ao tema, como o MEC, o CNPq, FINEP e a Capes.

O Funtec foi desenhado para atuar sobre um subsistema do CT\&l, qual seja, o que desenvolve pesquisa aplicada. Essencialmente compõem esse subsistema (que doravante denominaremos apenas como sistema) diversos atores:

- Instituições Tecnológicas (IT): são instituições públicas ou privadas sem fins lucrativos que tenham por missão executar atividades de pesquisa aplicada de caráter científico e tecnológico, bem como de desenvolvimento tecnológico. No sistema, sua função é executar projetos tecnológicos em parceria com empresas tendo objetivo de gerar novos produtos e processos que possam ser efetivamente introduzidos no mercado;

- Fundações de apoio à pesquisa e desenvolvimento (P\&D): são instituições cuja finalidade é dar suporte aos projetos de pesquisa de interesse das ITs públicas. No sistema, são responsáveis pelo gerenciamento dos recursos financeiros e dos contratos e acordos relativos aos projetos de pesquisa conduzidos por ITs públicas;

- Instituições financiadoras de projetos de pesquisa, desenvolvimento e inovação (PD\&I): são agentes responsáveis pela seleção, apoio financeiro e acompanhamento dos projetos de pesquisa aplicada conduzidos pelas ITs. Suas políticas e diretrizes funcionam como direcionadores da atuação das instituições clientes. Na esfera federal, as principais instituições financiadoras dos projetos cooperativos de PD\&I entre ITs e empresas são BNDES, FINEP, CNPq, e na estadual, destacam-se as Fundações Estaduais de Amparo à Pesquisa, tais como FAPESP, FAPER], FAPEMIG;

- Empresas: no Sistema, são aquelas que exercem atividade econômica diretamente relacionada ao escopo do projeto de PDEI. Com isso, agregam conhecimento sobre oportunidades e problemas do mercado-alvo e orientam o projeto considerando esse cenário econômico. Além disso, comprometem-se a introduzir no mercado a inovação gerada. A parceria com a IT 
ocorre em diferentes âmbitos, principalmente por meio de sua equipe de $P \& D$, que contribui tecnicamente no desenvolvimento do projeto, mas também por meio da equipe de marketing, que conhece o mercado-alvo, e por vezes inclui outros departamentos que contribuem em suas respectivas áreas de atuação, por exemplo, industrial e financeiro.

A principal interação com o ambiente externo se dá entre as empresas e o mercado, por meio do uso de soluções tecnológicas para inovar em seus processos e produtos, sendo que essa interação empresa/mercado pode ser intermediada pelas Fundações de Apoio. Assim, o propósito do Sistema em pauta é gerar desenvolvimento tecnológico e econômico para o país.

\section{O SISTEMA DE PESOUUISA APLICADA SUAS PERSPECTIVAS E A ATUAÇÃO DO FUNTEC}

O Funtec tem como principais funções promover a cultura de parceria entre instituições tecnológicas e empresas na condução de pesquisas aplicadas e produzir novas tecnologias que possam ser levadas ao mercado, e seu beneficiário direto de apoio são as Instituições Tecnológicas. O Sistema será examinado segundo três perspectivas: Competências; Processos Sistêmicos; e Desenvolvimento Tecnológico. Na Figura 1 a seguir está representada a ASE do Funtec, contendo suas perspectivas e objetivos. ${ }^{15}$ As subseções seguintes detalham, segundo essas óticas, como o sistema é caracterizado e o que o Funtec espera alcançar em cada nível.

\section{- FIGURA 1: ANÁLISE SISTÊMICA DE EFETIVIDADE DO FUNTEC}

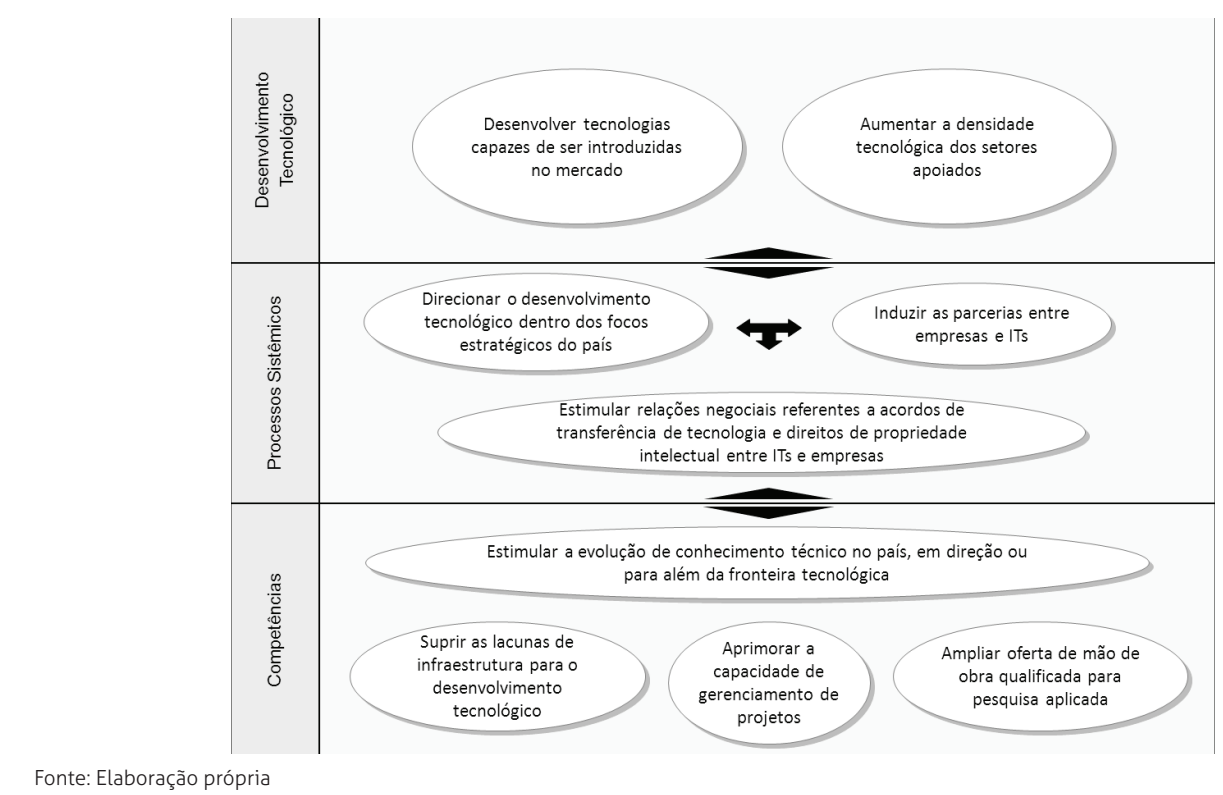

Revista Brasileira de Monitoramento e Avaliação | Número 8 | Julho-Dezembro de 2014 


\section{CRIAÇÃO E FORTALECIMENTO DE COMPETÊNCIAS}

As competências do Sistema de Pesquisa Aplicada podem ser classificadas em ativos tangíveis ou intangíveis. De uma forma geral, é possível agrupar a infraestrutura disponível para a realização da pesquisa como a competência tangível.

No entanto, por se tratar de um sistema intensivo em conhecimento, as competências intangíveis são fundamentais e contemplam capacitações técnicas em atividades de P\&D, gerenciais na gestão de projetos, e mercadológicas para que a pesquisa tenha real potencial de ser introduzida no mercado.

Para a reunião de todos esses ativos, é necessária a parceria, pelo menos, entre IT e empresa: aquela participa com suas competências técnicas e seus laboratórios, enquanto esta aporta suas competências mercadológicas e técnicas. Já as gerenciais podem ser aportadas pela Fundação de Apoio e, na sua ausência, pela IT e pela empresa.

Para que o sistema seja reforçado nessa perspectiva, o resultado esperado do apoio do Funtec é o fortalecimento das respectivas competências de cada um de seus atores. Espera-se que esse apoio, conforme apresentado na perspectiva inferior da Figura 1, contribua para suprir as lacunas de infraestrutura para o desenvolvimento tecnológico; estimule a evolução do conhecimento técnico em direção, ou até mesmo além, da fronteira tecnológica; amplie a oferta de mão de obra qualificada para pesquisa aplicada no país; e aprimore a capacidade dos atores do sistema de gerenciar projetos de PD\&l.

\section{MELHORIA DE PROCESSOS SISTÊMICOS}

Na perspectiva de processos deve ser examinada a natureza das relações entre seu ator central, a instituição tecnológica, e os outros agentes que o compõe.

Os processos que promovem a realização da pesquisa aplicada podem ser decompostos para fins de análise. Assim, as parcerias entre ITs podem se originar nas relações de trabatho e rotatividade de seus pesquisadores, na participação em seminários e congressos, e na produção científica publicada. Já a cooperação da empresa com a Instituição pode ocorrer a partir da formação ou especialização de funcionários das empresas nas ITs, da contratação de serviços tecnológicos e consultorias da IT e também pelo encontro em eventos técnicos e pela produção científica dos pesquisadores da instituição e da empresa. Já a relação da Instituição Tecnológica com o governo, com as agências reguladoras e de fomento podem se dar em duas direções. Esses entes públicos podem consultá-la para formular suas políticas e normas. Por outro lado, as normas e políticas implementadas estimularão e direcionarão as pesquisas realizadas pelas ITs. Uma análise similar pode ser feita para a interação das empresas com os entes públicos.

Para que esse sistema se desenvolva e funcione cada vez mais coordenado, o Funtec atua com os objetivos de induzir a parceria entre ITs e empresas; direcionar os focos das pesquisas para os temas prioritários definidos pelas políticas públicas; e, por fim, estimular relações negociais na distribuição dos direitos de propriedade intelectual gerados 
em projetos cooperativos entre ITs e empresas. Esses objetivos estão apresentados na perspectiva intermediária da Figura 1.

\section{PROMOÇÃO DO DESENVOLVIMENTO TECNOLÓGICO}

Para que ocorra o desenvolvimento tecnológico no país, é necessário que as ITs reconheçam as oportunidades contidas nas diferentes trajetórias tecnológicas dos setores priorizados, segundo uma visão empresarial, e as transformem em novos produtos e processos.

Não é que as ITs conheçam ou saibam as demandas de novas tecnologias a priori, mas sim que possam captar essas oportunidades tecnológicas, segundo uma leitura do mercado, das políticas públicas, das rotas tecnológicas existentes e do potencial de desenvolvimento econômico para o país, transformando-as em potenciais inovações.

Ao selecionar projetos cooperativos entre ITS e empresas com características que demonstrem sua aderência ao potencial de desenvolvimento tecnológico e econômico de determinado setor, o Funtec espera contribuir para o reposicionamento competitivo da indústria brasileira. Assim, conforme apresentado na perspectiva superior da Figura 1, tem como objetivos o desenvolvimento de tecnologias capazes de serem introduzidas no mercado, e o aumento da densidade tecnológica nos setores selecionados.

\section{Conclusão}

A aplicação da ASE no caso Funtec demonstrou vantagens em relação ao uso do QL $\mathrm{L}$. abordagem metodológica da ASE foi construída para permitir o M\&A de intervenções estruturantes, nas quais os efeitos esperados são principalmente sistêmicos, ou seja, ultrapassam os objetivos declarados e contribuem com outras políticas públicas. Com isso, foi preciso definir, em primeiro lugar, o sistema em que a intervenção vai operar, induzindo a equipe formuladora ao conhecimento/ diagnóstico sobre o ambiente de atuação do programa. A seguir esse sistema é visto por meio de suas várias perspectivas: no caso do Sistema de Pesquisa Aplicada elas são Competências, Processos e Desenvolvimento Tecnológico. Assim, a ASE permite a definição dos vários efeitos sistêmicos da intervenção e dos seus indicadores, lançando as bases para o M\&A de seus resultados. Assim se tem uma visão bem mais ampla, não se restringindo apenas ao que seria o objetivo declarado do programa.

Por se tratar de uma nova metodologia para o M\&A de intervenções estruturantes, algumas questões precisam ganhar maturidade. Por exemplo, o critério para aplicação da metodologia ASE ainda está situado no campo subjetivo, qual seja, a classificação de programas finalísticos ou estruturantes. Outro ponto é que ainda não está claro qual é o grau de profundidade necessário na definição do sistema

Revista Brasileira de Monitoramento e Avaliação | Número 8 | Julho-Dezembro de 2014 
para que sejam explicitados os objetivos do programa.

Entretanto considerou-se que com a experimentação do método, essas questões serão esclarecidas. Essa metodologia propiciou as bases para que seja possível um adequado monitoramento futuro dos efeitos sistêmicos do programa, possibilitando, posteriormente, uma avaliação de qualidade. Assim será possível uma visão mais realista das vantagens da ASE.

\section{- ANEXO A: INDICADORES POR CADA OBJETIVO DA ASE DO FUNTEC}

\section{OBJETIVOS}

\section{INDICADORES}

$1.1 N^{\circ}$ de publicações técnicas relevantes

1. Estimular a evolução de conhecimento técnico no país, em

direção ou para além da fronteira tecnológica

1.2 Capacitação técnica gerada para ITs ou empresas

2. Suprir as lacunas de infraestrutura para o desenvolvimento 2.1 Valor e $n^{\circ}$ de novos laboratórios construídos tecnológico

2.2 Valor e $n^{\circ}$ de laboratórios modernizados

3. Aprimorar a capacidade de gerenciamento de projetos

$3.1 \%$ de valor e atraso na conclusão dos projetos

4. Ampliar oferta de mão de obra qualificada para pesquisa 4.1 No de pessoas qualificadas e por nível de ensino nas equipes aplicada apoiadas

5. Induzir as parcerias entre empresas e ITS

5.1 Pesquisa de Percepção

6. Direcionar o desenvolvimento tecnológico dentro dos focos estratégicos do país, segundo o Funtec

6.1 Pesquisa de Percepção

7. Estimular relações negociais referentes a acordos de trans-

ferência de tecnologia e direitos de propriedade intelectual 7.1 Taxa de conflito daqueles com acordo firmado entre ITs e empresas

8.1 Taxa de conclusão do desenvolvimento tecnológico

8.2 Taxa de tecnologias introduzidas no mercado

8. Desenvolver tecnologias capazes de ser introduzidas no

mercado

8.3 Taxa de tecnologias com previsão de introdução no mercado

$8.4 \mathrm{~N}^{\circ}$ de patentes depositadas

9. Aumentar a densidade tecnológica dos setores apoiados

9.1 Participação de produtos novos na receita total das empresas apoiadas 


\section{Referências bibliográficas}

BERTALANFFY, L. Von. General System Theory: foundations, development, applications. Nova York: George Braziller, 1969.

BRASIL. Técnicas de auditoria: marco lógico. Brasília: TCU, 2001.

CASSIOLATO, M.; GUERESI, S. Como elaborar Modelo Lógico: roteiro para formular programas e organizar avaliação. Brasília: IPEA, 2010.

EARL, S.; CARDEN, F.; SMUTYLO, T. Outcome Mapping: building learning and reflection into development programs. Ottawa: International Development Research Centre, 2001.
KAPLAN, R. S.; NORTON, D. P. Mapas Estratégicos - Balanced Scorecard: convertendo ativos intangíveis em resultados tangíveis. Rio de Janeiro: Elsevier, 2004.

KUNZ, H.; SCHAAF, T. General and specific formalization approach for a Balanced Scorecard: an expert system with application in health care. Expert Systems with Applications, [S. L.], v. 38, p. 1947-1955. 2011.

PFEIFFER, P. O quadro lógico: um método para planejar e gerenciar mudanças. Revista do Serviço Público, Brasília, v. 51, n. 1, p. 81-102. 2000. 